\title{
Facile Method for Preparation of Silica Coated Monodisperse Superparamagnetic Microspheres
}

\author{
Xuan-Hung Pham, ${ }^{1}$ San Kyeong, ${ }^{2}$ Jaein Jang, ${ }^{3}$ Hyung-Mo Kim, ${ }^{1}$ Jaehi Kim, ${ }^{2}$ \\ Seunho Jung, ${ }^{1}$ Yoon-Sik Lee, ${ }^{2}$ Bong-Hyun Jun, ${ }^{1}$ and Woo-Jae Chung ${ }^{3}$ \\ ${ }^{1}$ Department of Bioscience and Biotechnology, Konkuk University, Seoul 143-701, Republic of Korea \\ ${ }^{2}$ School of Chemical and Biological Engineering, Seoul National University, Seoul 151-747, Republic of Korea \\ ${ }^{3}$ Department of Genetic Engineering, College of Biotechnology and Bioengineering, Sungkyunkwan University, \\ Suwon 440-746, Republic of Korea
}

Correspondence should be addressed to Bong-Hyun Jun; bjun@konkuk.ac.kr and Woo-Jae Chung; wjchung@skku.edu

Received 15 October 2015; Revised 28 December 2015; Accepted 10 January 2016

Academic Editor: Zhisong Lu

Copyright ( $\odot 2016$ Xuan-Hung Pham et al. This is an open access article distributed under the Creative Commons Attribution License, which permits unrestricted use, distribution, and reproduction in any medium, provided the original work is properly cited.

\begin{abstract}
This paper presents a facile method for preparation of silica coated monodisperse superparamagnetic microsphere. Herein, monodisperse porous polystyrene-divinylbenzene microbeads were prepared by seeded emulsion polymerization and subsequently sulfonated with acetic acid/ $\mathrm{H}_{2} \mathrm{SO}_{4}$. The as-prepared sulfonated macroporous beads were magnetized in presence of $\mathrm{Fe}^{2+} / \mathrm{Fe}^{3+}$ under alkaline condition and were subjected to silica coating by sol-gel process, providing water compatibility, easily modifiable surface form, and chemical stability. FE-SEM, TEM, FT-IR, and TGA were employed to characterize the silica coated monodisperse magnetic beads $(\sim 7.5 \mu \mathrm{m})$. The proposed monodisperse magnetic beads can be used as mobile solid phase particles candidate for protein and DNA separation.
\end{abstract}

\section{Introduction}

Manipulation of biological objects at the cellular scale is necessary for both fundamental studies and the purification of biological products. For example, optical tweezers $[1,2]$ allow studying the strength of interactions between single molecules with piconewton accuracy such as DNA and protein. Due to the advantage of noncontact with biomolecules or cell during separation, the magnetic manipulation has been attracted in bioapplication over the several decades [39].

The dimension of the magnetic nano-/microparticles, the key factor for the successful magnetic separation, is usually less than $10 \mu \mathrm{m}$ to ensure perfect and selective interaction with biological objects. These particles' surface must be modified with biocompatible inorganic or organic materials to both prevent aggregation caused by hydrophobic or magnetic attraction and facilitate the immobilization of specific ligands such as antibody, aptamer, and DNA $[3,10]$.
The magnetic microparticles have been attracted for use in bioapplication for many years, due to the advantages that they offer easily scalable, time-efficient, cost-effective, and gentle separation of biological compounds by using external magnetic field gradient [11-17]. The magnetic microspheres offered many exciting applications in biomedical field as a solid support for protein purification [18-20], targeted drug delivery [21-24], cell separation [25-27], medical diagnosis [28-30], immunoassays [31], DNA sequencing [32], and cell analysis [33]. Various materials have been used for matrices embedding superparamagnetic nanoparticles in the form of microbeads including mesoporous silica, polysaccharides, poly(ethylene glycol), and many other synthetic polymers [26, 34-39]. By doing so, the resulting microbeads retain superparamagnetic property with tunable size.

The porous PS-DVB (polystyrene-divinylbenzene) beads usually give a very rigid structure resulting from their highly cross-linked matrix and, thus, they had less possibility of collapsing in most solvents, which allows the solvents and 
reagents to freely access the internal reactive sites through the interconnected pore networks [40-43]. The microbeads with pore networks can act as monodisperse core structures and provide the space within the beads where in situ forming nanomagnetite resides. Recently, we reported on the use of porous PS-DVB based monodisperse magnetic beads with SERS (surface-enhanced Raman scattering) active property (magnetic SERS beads) in protein separation [44, 45].

Despite extensive efforts made by many research groups, the demand for magnetic polymeric particles having uniform size, superparamagnetic property, chemical stability, userdefined magnetic contents, biocompatibility, and the readily tailorable surface is still increasing in potential application to biochips and bioseparation. In this paper, we demonstrate our strategy to prepare monodisperse porous PS-based superparamagnetic beads $(\sim 7.5 \mu \mathrm{m})$, which on surface were coated with silica that can result in improving biocompatibility and capability for facile surface modification [46-48].

\section{Experimental}

2.1. Materials. Tetraethylorthosilicate (TEOS), ammonium hydroxide $\left(\mathrm{NH}_{4} \mathrm{OH}\right)$, styrene, 2,2' -azobis-isobutyronitrile (AIBN), dibenzoyl peroxide (BPO, including 97\% of active compound), dibutyl phthalate (DBP), sodium silicate, $\mathrm{Fe}_{3} \mathrm{Cl}_{3} \cdot 6 \mathrm{H}_{2} \mathrm{O}, \mathrm{FeCl}_{2} \cdot 4 \mathrm{H}_{2} \mathrm{O}$, ethylene glycol, $N, N$-dimethylformamide (DMF), sodium dodecyl sulfate (SDS), polyvinylpyrrolidone-40 (PVP-40, Mr: 40,000), N,N-diisopropylethylamine (DIEA), and (3-aminopropyl)triethoxysilane (APTS) were purchased from Sigma-Aldrich (MO, USA).

\subsection{Methods}

2.2.1. Preparation of Monodisperse Macroporous PS-DVB Beads. Monodisperse macroporous PS-DVB (polystyrenedivinylbenzene) beads were prepared by seeded polymerization methods. Monodisperse PS seeds $(4 \mu \mathrm{m})$ were prepared using dispersion polymerization method [49]. Dispersion medium was ethanol/2-methoxyethanol $(3: 2)$ which contains $1 \mathrm{~g}$ of PVP-40 as a steric stabilizer $(90 \mathrm{~mL})$. AIBN $(150 \mathrm{mg})$ was dissolved in styrene $(15 \mathrm{~mL})$ in which inhibitor was removed and then was added to the as-prepared dispersion medium. After sonication for $10 \mathrm{~min}$, dispersion polymerization was performed in a cylindrical reaction chamber with shaking $(120 \mathrm{cpm})$ at $70^{\circ} \mathrm{C}$ for $20 \mathrm{~h}$. The suspension was centrifuged and the precipitates were washed intensively with distilled water by repeating centrifugation and vortexing. Finally, the PS seeds were washed with ethanol and vacuumdried overnight affording PS seeds ( $4 \mu \mathrm{m}, 8.3 \mathrm{~g})$.

Swelling and polymerization processes were performed in a glass reactor equipped with overhead stirrer and a reflux condenser in an oil bath equipped with a temperature controller. The two-step seeded polymerization method was employed for preparation of monodisperse macroporous poly(styrene-co-DVB) (PS-DVB) beads. The PS seeds $(4 \mu \mathrm{m}$, $700 \mathrm{mg})$ were dispersed in DBP $(0.7 \mathrm{~mL})$ emulsified aqueous medium $(100 \mathrm{~mL})$ containing $0.25 \%$ (w/w) SDS. The resultant dispersion medium was stirred $(400 \mathrm{rpm})$ at room temperature for $20 \mathrm{~h}$ for swelling the PS seeds with DBP.
Mixture of styrene $(4.6 \mathrm{~mL})$ and DVB $(2.3 \mathrm{~mL})$ in which BPO (240 mg) was dissolved was emulsified in an aqueous medium $(100 \mathrm{~mL})$ containing $0.25 \%(\mathrm{w} / \mathrm{w})$ SDS by using homogenizer for $1 \mathrm{~min}$. The emulsified monomer solution was added to the DBP-swollen PS seeds dispersion medium being stirred. Monomer swelling was performed for $20 \mathrm{~h}$ at room temperature with continuous stirring (400 rpm). After swelling process, an aqueous solution of $10 \%(\mathrm{w} / \mathrm{v})$ PVA in deionized water $(10 \mathrm{~mL})$ was added to the dispersion medium and the medium was purged with nitrogen stream for $30 \mathrm{~min}$. The seeded polymerization was performed at $70^{\circ} \mathrm{C}$ for $20 \mathrm{~h}$ with continuous stirring $(200 \mathrm{rpm})$ to obtain monodisperse PS-DVB beads. The polymer beads were intensively washed with warm deionized water $\left(50^{\circ} \mathrm{C}\right)$ by repeating washing and centrifugation. Subsequently, the collected beads were washed with ethanol and THF intensively to remove DBP and linear polymer. Finally, the beads were dried in vacuum at $30^{\circ} \mathrm{C}$ for $24 \mathrm{~h}$ to obtain macroporous PS-DVB beads $(7.5 \mu \mathrm{m}$, $2.5 \mathrm{~g})$.

2.2.2. Sulfonation of Macroporous PS-DVB Beads. Sulfonation of the PS-DVB beads was performed with reference to the reported method [50]. Briefly, monodisperse PS-DVB beads $(1 \mathrm{~g})$ were added to $5 \mathrm{~mL}$ of acetic acid in an ice bath. Sulfuric acid $(50 \mathrm{~mL})$ was then slowly added to the beads at room temperature and the temperature was increased up to $90^{\circ} \mathrm{C}$ and the resin mixture was stirred for $30 \mathrm{~min}$ to $2 \mathrm{hr}$. The dispersion was poured into iced water $(400 \mathrm{~mL})$ to quench the reaction and the sulfonated PS-DVB beads were collected by centrifugation. The beads were extensively washed with deionized water by repeating centrifugationdecantation. Subsequently, the sulfonated microspheres were washed three times with ethanol and dried under vacuum (1.1 g).

\subsubsection{Magnetization of Sulfonated Macroporous PS-DVB} Beads. The sulfonated macroporous PS-DVB beads $(500 \mathrm{mg})$ were dispersed in deionized water $(10 \mathrm{~mL})$ at room temperature with mechanical stirring $(200 \mathrm{rpm})$ and purging with nitrogen. A freshly prepared mixture of $\mathrm{FeCl}_{3} \cdot 6 \mathrm{H}_{2} \mathrm{O}$ (618 mg, $2.26 \mathrm{mmol}$ ) and $\mathrm{FeCl}_{2} \cdot 4 \mathrm{H}_{2} \mathrm{O}(257 \mathrm{mg}, 1.28 \mathrm{mmol})$ in deionized water $(10 \mathrm{~mL})$ was added to the dispersion for adsorption. After $2 \mathrm{~h}$, with continuous stirring $28 \%$ ammonium hydroxide $(50 \mathrm{~mL})$ was added dropwise to the beads suspension for $40 \mathrm{~min}$. The magnetized microspheres were isolated from the mixture by centrifugation and briefly washed with $25 \%$ TFA and then extensively with deionized water and ethanol. Finally, the magnetized microspheres were dried under vacuum (magnetized microspheres, $7.5 \mu \mathrm{m}$, $653 \mathrm{mg}$ ).

2.2.4. Preparation of Silica Coated Magnetic Beads. APTS solution $(1 \%(\mathrm{v} / \mathrm{v}), 100 \mathrm{~mL})$ was added to $100 \mathrm{mg}$ of magnetic sulfonated PS-DVB beads and was shaken for $10 \mathrm{~min}$ at room temperature. Thereafter, ammonium hydroxide $(28 \%$, $2 \mathrm{~mL}$ ) was added to the bead dispersion and was shaken for $20 \mathrm{~min}$ at room temperature. To the dispersion TEOS $(2 \mathrm{~mL})$ was added and vigorously shaken for $12 \mathrm{~h}$ at room 


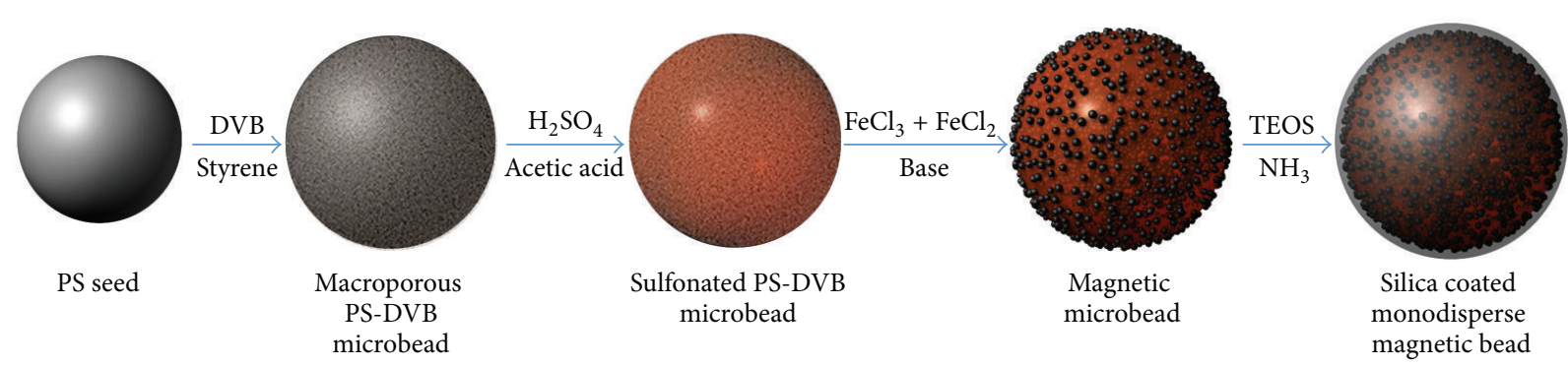

Scheme 1: Synthetic scheme of silica coated superparamagnetic microbead.

temperature. The resulting beads (silica coated magnetic beads) were collected by magnet and washed with ethanol five times.

2.2.5. Streptavidin Separation of Silica Coated Magnetic Beads. APTS $(240 \mu \mathrm{L})$ and ammonium hydroxide $(40 \mu \mathrm{L})$ were added to $4 \mathrm{~mL}$ absolute ethanol containing $200 \mathrm{mg}$ of silica coated magnetic beads and incubated for $6 \mathrm{~h}$ at room temperature. The resulting beads (amine-silica coated magnetic beads) were collected by magnet, washed with ethanol five times, and dispersed into PBS, pH 7.2, to obtain $10 \mathrm{mg} / \mathrm{ml}$ of amine-silica coated magnetic beads.

Biotin (5 mg) was activated with EDC (40 mg) and NHS $(20 \mathrm{mg})$ in PBS, $\mathrm{pH} \mathrm{7.2,} \mathrm{for} 1 \mathrm{~h}$ at room temperature. Then, this solution was added into amine-silica coated magnetic beads $(1 \mathrm{mg}, 100 \mu \mathrm{L})$ to modify biotin on its surface. The biotinylated silica coated magnetic beads were collected by magnet, washed with ethanol five times, and dispersed into $100 \mu \mathrm{L}$ PBS, pH 9.0. The biotinylated silica coated magnetic beads were passivated with 5\% BSA in PBS, $\mathrm{pH}$ 9.0, for preventing nonspecific binding.

Streptavidin-FITC $(100 \mu \mathrm{g})$ in PBS pH 9.0 was added into the biotinylated silica coated magnetic beads and incubated for $30 \mathrm{mins}$ at room temperature. The biotinylated silica coated magnetic beads after incubation with streptavidinFITC were collected by magnet, washed with PBS, $\mathrm{pH}$ 9.0, containing $0.1 \%$ Tween 20 for five times, and dispersed into $100 \mu \mathrm{L}$ PBS, pH 9.0. The fluorescent images were collected by the Olympus BX-UCB at room temperature with $200 \mathrm{~ms}$ exposure.

2.2.6. Characterization. Cross-sectional images of the beads were obtained using transmission electron microscopy (TEM, Jeol Inc., JEM 1010). The slices for the TEM analysis were prepared by means of an ultramicrotome (RCM, MTX). The surface and cross-sectional images of the beads were obtained using field emission scanning electron microscopy (FE-SEM, Carl Zeiss, SUPRA 55VP). The existence of the magnetic NP particles was confirmed by means of an energy dispersive X-ray spectrometer (EDX, Bruker, EDX). The amount of magnetic particles embedded in the beads was measured by a thermogravimetric analyzer (TGA, PerkinElmer Pyris 6).

\section{Results and Discussion}

For preparing magnetic beads, we employed a method using magnetization of uniform porous microbeads. As shown in Scheme 1, monodisperse superparamagnetic microbead has a spherical core-shell structure with silica layer over magnetized core PS-DVB bead.

Monodisperse polystyrene-divinylbenzene (PS-DVB) beads $(7.5 \mu \mathrm{m}$, cross-linked with $33 \% \mathrm{DVB})$ were prepared using the seeded polymerization method that was previously reported by Ugelstad [49]. The seeded polymerization is a useful method for preparation of monodisperse microbeads which is essential for bioapplications including relative quantitative flow cytometry and biochip applications. The PS seeds were prepared in the range of $4.0 \sim 4.2 \mu \mathrm{m}(61 \%$ solid yield) (Figure 1(a)) and the molecular weight was 29,000 (determined by GPC analysis). The final PS-DVB beads were prepared with repolymerization of the seeds $(4 \mu \mathrm{m}$, $2.5 \mathrm{~g}, 47 \%$ solid yield) using DBP as a porogen and activated swelling agent.

The prepared PS-DVB microbeads showed a macroporous surface (Figure 1(b)), which enables the magnetite particles to form easily inside the microbeads upon magnetization from iron salts adsorbed in the porous structures. However, the PS-DVB microbead itself was not compatible in aqueous solution to adsorb the ferrous and ferric salts. Thus, we introduced sulfonate groups onto the PS-DVB microbeads to make them compatible in aqueous solution and the sulfonate group might have the effect that the iron salt is drawn into the microbeads and is considerably bound therein.

Sulfonated PS-DVB polymers have been used for many years in cationic exchange chromatography, as well as other analytes [51-53]. Recently, Chambers and Fritz have reported on the simple preparation of sulfonated PS-DVB with the $-\mathrm{SO}_{3} \mathrm{H}$ loading range of $0.27 \sim 2.63 \mathrm{mmol} / \mathrm{g}$ using sulfonic acid and acetic acid [50]. To demonstrate the effect of sulfonation loading level of beads to magnetization, various loading levels of sulfonate group were prepared by controlling temperature and reaction time (data not shown). As a result from applying Chamber's method to the as-prepared PSDVB microbeads, we obtained high loaded sulfonated PSDVB (PS-DVB-SO ${ }_{3} \mathrm{H}$ ) of $4.1 \mathrm{mmol} / \mathrm{g}$ by elemental analysis. FT-IR analysis showed that the sulfonation of the phenyl groups of the PS-DVB was successfully achieved (Figure 2). In the FT-IR spectra of PS-DVB before (Figure 2(a)) and 


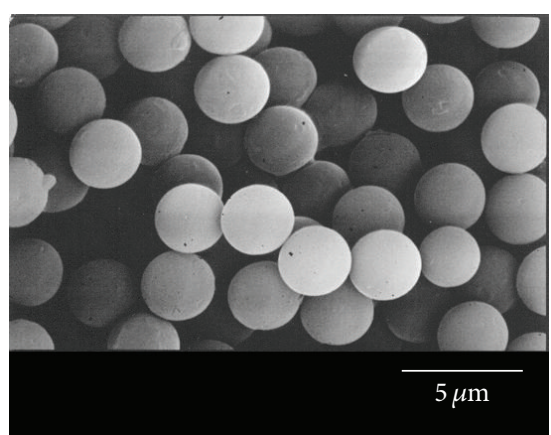

(a)
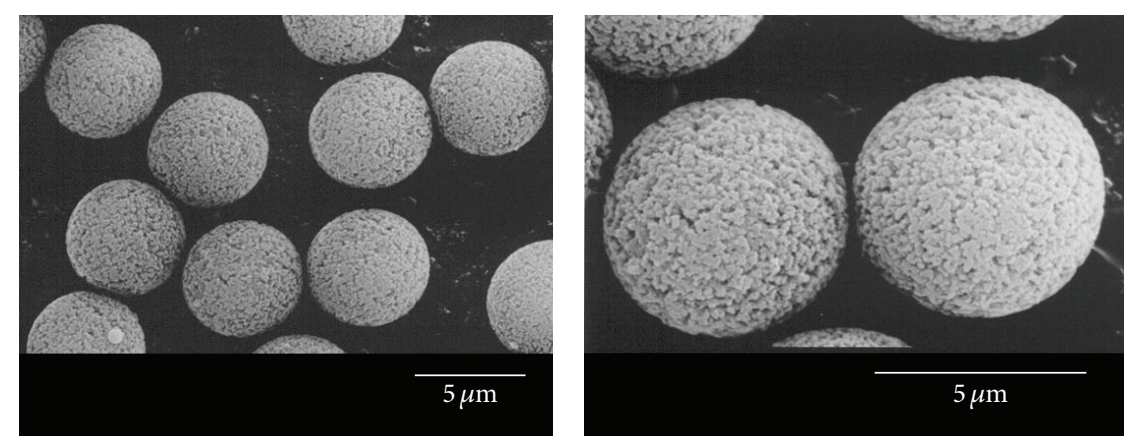

(b)

FIGURE 1: SEM images of (a) PS seeds and (b) PS-DVB microbeads prepared by seeded emulsion polymerization at low magnification (left) and high magnification (right).

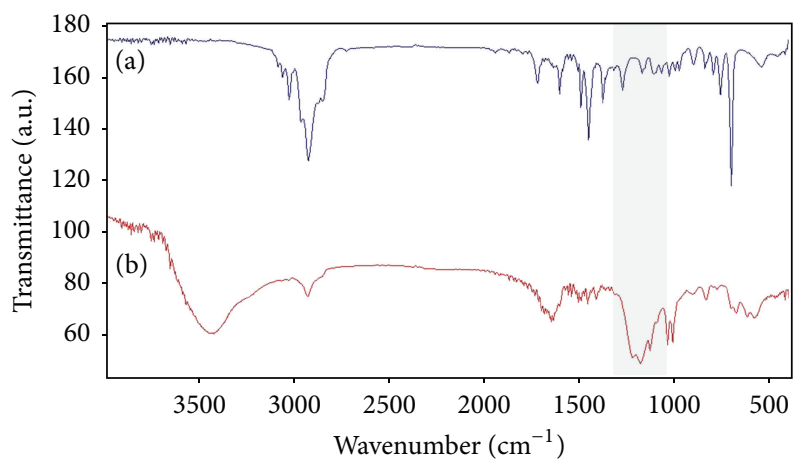

FIGURE 2: FT-IR spectra of PS-DVB microbeads (a) before and (b) after sulfonation. Shaded area represents the characteristic peak of sulfonate groups. Resolution setting, $4 \mathrm{~cm}^{-1}, 16$ scans.

after sulfonation (Figure 2(b)), the changes in the combination vibrations (finger bands) between 1950 and $1650 \mathrm{~cm}^{-1}$ particularly characterize the phenyl group in the microbeads. The band centered around $1200 \mathrm{~cm}^{-1}$ (Figure 2(b)) represents the characteristic peak of the $\mathrm{O}_{y} \mathrm{~S}_{y} \mathrm{O}$ asymmetric stretching vibration. The absorbance peaks at 1005 and $1126 \mathrm{~cm}^{-1}$ result from the vibrations of phenyl ring substituted with a sulfonate group and sulfonate anion, respectively, that are attached to phenyl ring [54]. A broad band centered around $3450 \mathrm{~cm}^{-1}$ is associated with $\mathrm{OH}$ stretching vibration of the sulfonic acid group (Figure 2(b)).

Direct polymerization of ion-binding monomers during the seeded emulsion polymerization process can be an alternative method of preparing functional macroporous microbeads. However, formation of uniformly macroporous structures using the ionic monomers is often challenging. In contrast, the postmodification of highly cross-linked macroporous beads as described above can preserve the structure of monodisperse microbeads.

After magnetization of sulfonated PS-DVB microbeads with a mixture of ferrous ions and ferric ions (2:1 molar ratio) in the excess basic solution of ammonium hydroxide, precipitates of magnetite were observed in the solution as well as in the beads as they turned blackish. The surface morphology of microbeads was not significantly changed after magnetization, meaning that the magnetite was formed and precipitated mostly inside the porous structure of the microbeads (Figures 3(a) and 3(b)). After purifying the magnetized beads from the mixture by centrifugation, we confirmed that the precipitate is indeed iron oxide from energy dispersive X-ray spectroscopy (Figure 3(c)). These magnetized microbeads indeed responded to external magnetic field (Figure 3(d), inset). The magnetic property was maintained even after keeping the beads for $4 \mathrm{~h}$ in $100 \%$ TFA.

The saturation magnetization value of the resulting beads was $7.6 \mathrm{emu} / \mathrm{g}$ (Figure 3(d)), which is relatively lower than that of commercial magnetites $(\sim 70 \mathrm{emu} / \mathrm{g})$. However, the magnetized beads showed strong magnetic property enough in that they can be separated from the solvent by 4,000 gauss magnet in $4 \mathrm{sec}$. The magnetized sulfonated PS-DVB microbeads consist of many isolated, around a few tens $\mathrm{nm}$, iron oxide grains that are embedded in a polymer matrix which can lead to their superparamagnetic behavior. The sulfonation of the beads and the subsequent magnetization successfully provided a means for preparing magnetic microbeads in straightforward and cost-effective way.

Iron oxide contents of the magnetized beads were measured by TGA (Figure 4). Iron oxide content was in the range of $18-26 \%$ whose value was suitable for further application of the magnetic beads comparing with the conventional magnetic beads. In the magnetization process, we attempted to simplify the purification by washing the beads after iron salts adsorption to prevent the formation of the magnetite in the solution after precipitation of iron salts using ammonium hydroxide (MAG 1). The iron oxide content of the magnetized beads was lower than that of beads prepared by in situ precipitation method without additional washing step (MAG 2).

Finally, the magnetic beads were coated with a silica shell using APTS and tetraethoxyorthosilicate (TEOS) for their surface to be water-compatible and amenable to chemical modification using various types of functional silane compounds. The amino silane compound APTS is prone to promote the adhesion of silane layer on the negatively charged sulfonated beads, thereby facilitating the further basecatalyzed condensation of TEOS from the surface. We found 


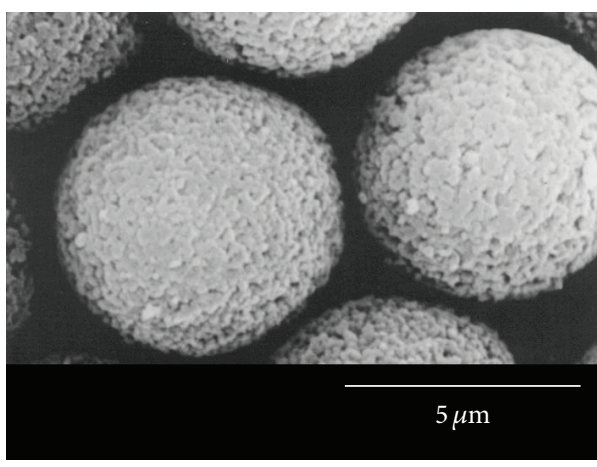

(a)

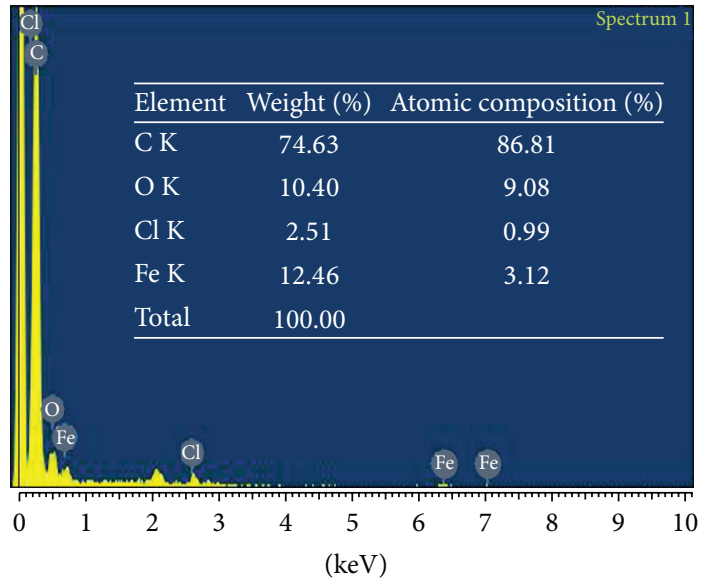

Full scale 782 cts cursor: $0.000 \mathrm{keV}$

(c)

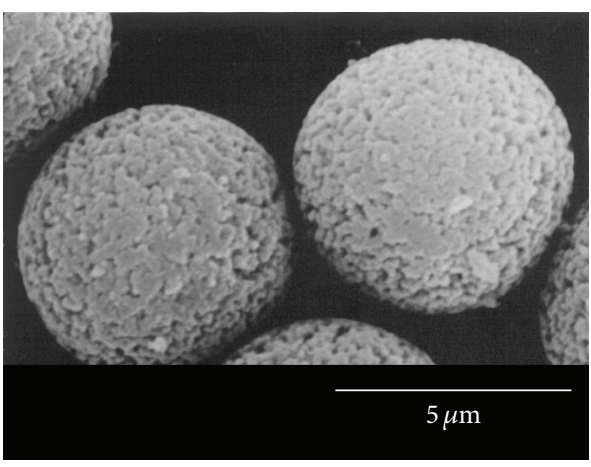

(b)

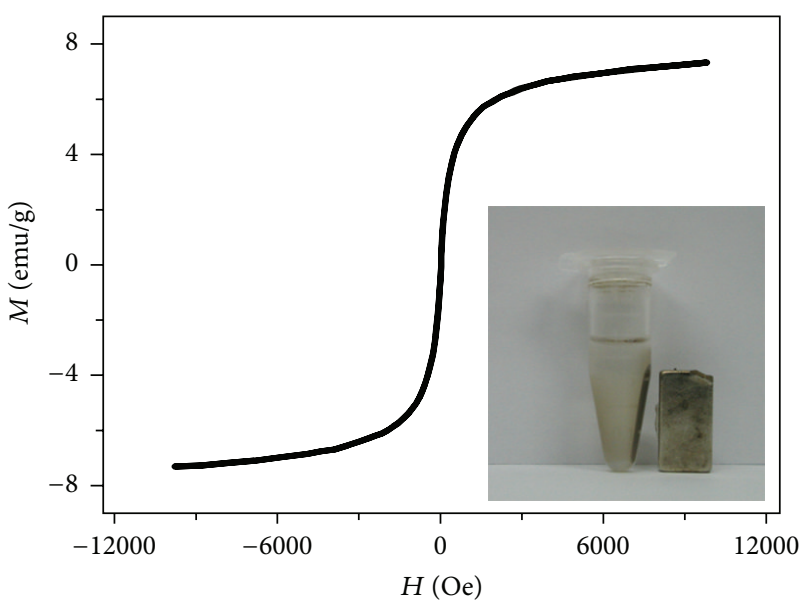

(d)

FIGURE 3: Characteristics of magnetized sulfonated microbeads. SEM images of sulfonated PS-DVB microbeads (a) before and (b) after magnetization. (c) Representative EDS analysis result of magnetized microbeads. (d) Hysteresis loops of magnetic beads. Inset: the beads were attracted to one side of the tube using a magnet.

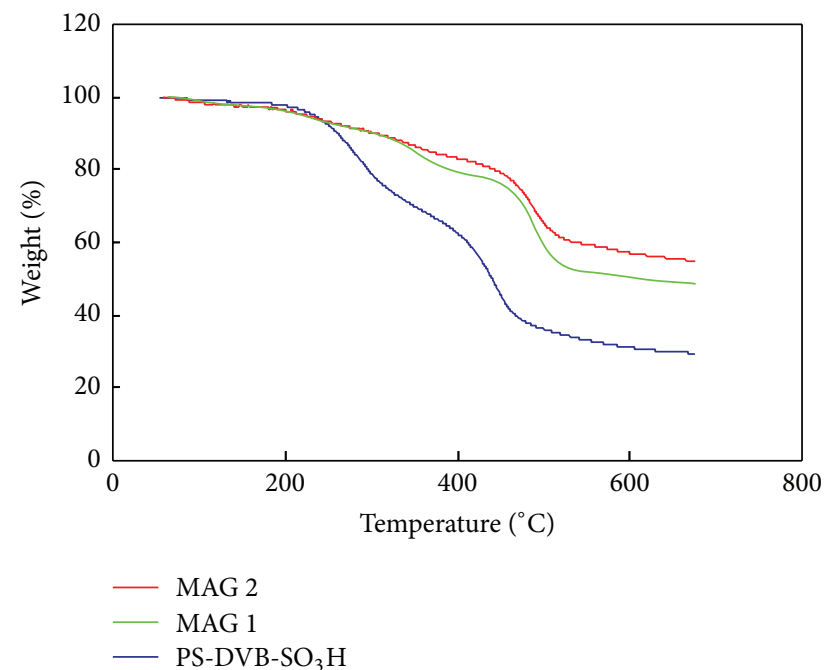

FIGURE 4: TGA thermograms of sulfonated PS-DVB microbeads (PS-DVB-SO $\mathrm{S}_{3} \mathrm{H}$ ) before and after magnetization: (MAG 1) magnetization after washing the iron salt adsorbed beads; (MAG 2) magnetization without washing (in situ magnetization); iron oxide content: 18\% (MAG 1) and 26\% (MAG 2). that this pretreatment greatly enhanced the silica coating efficiency as compared to no pretreatment (data not shown) and pretreatment with (3-mercaptopropyl)triethoxysilane solution [44]. The surface morphology of the beads significantly changed after the sol-gel process (Figures 5(a)$5(d)$ ) revealing formation of silica grains on the surface (Figures 5(b) and 5(d)). From the cross-sectional analysis, the silica shell of 200-300 nm was observed around the core magnetic beads. The silica coated magnetic beads were also homogeneous in size and could be obtained in gram scale.

To support the presence of silica coating on the magnetic beads, we carried out EDX analysis of the sulfonated PSDVB, magnetic PS-DVB, and silica coated magnetic PS-DVB beads. The result was shown in Figure 6. The sulfonated PS-DVB beads contained $\mathrm{C}, \mathrm{O}$, and $\mathrm{S}$ elements with the atomic composition of $86.5 \%, 9.1 \%$, and $4.4 \%$, respectively, in Figure 6(a). After magnetic nanoparticles were deposited on the sulfonated PS-DVB beads, C element decreased dramatically to $67.2 \%$ while O element increased up to $20.3 \%$. Particularly, the presence of Fe element with 5.7\% confirmed the successful deposition of magnetic nanoparticles on the sulfonated PS-DVB beads. When the silica shell was coated 


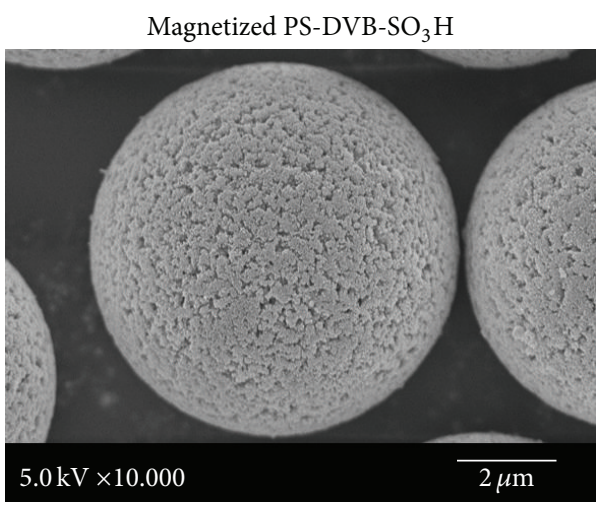

(a)

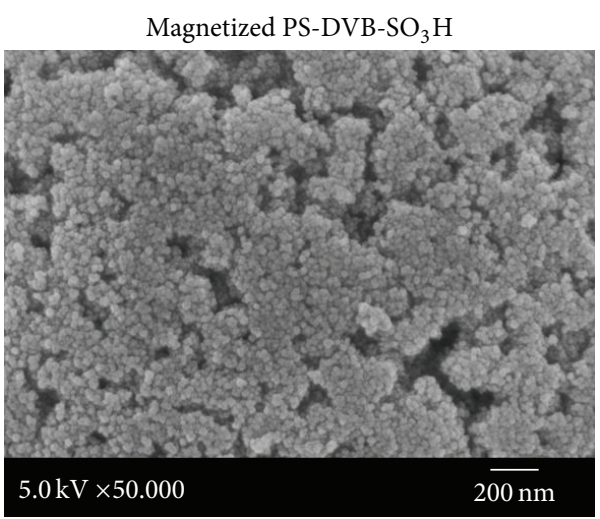

(c)

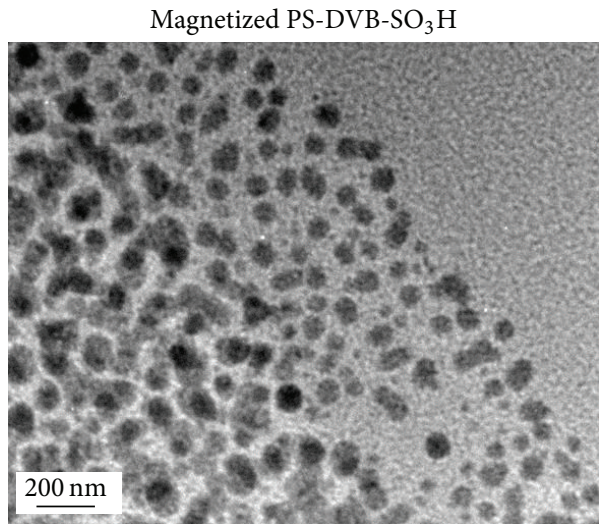

(e)

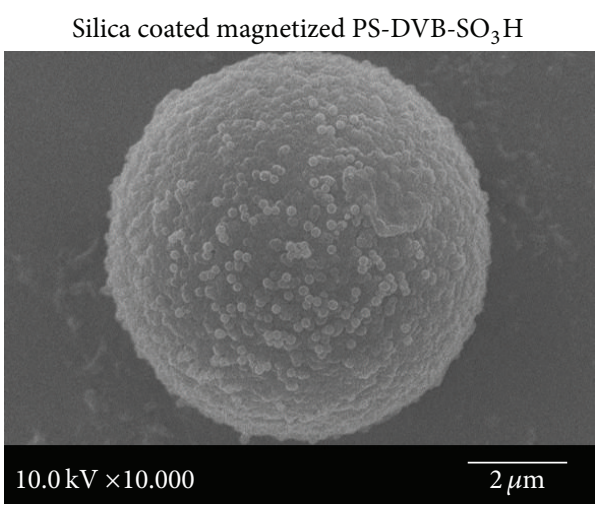

(b)

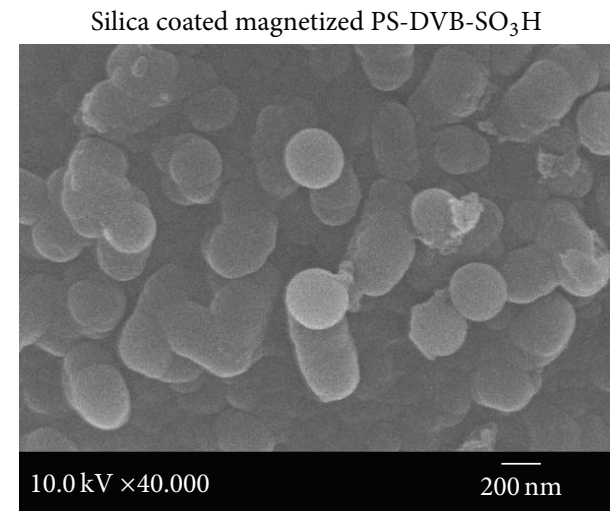

(d)

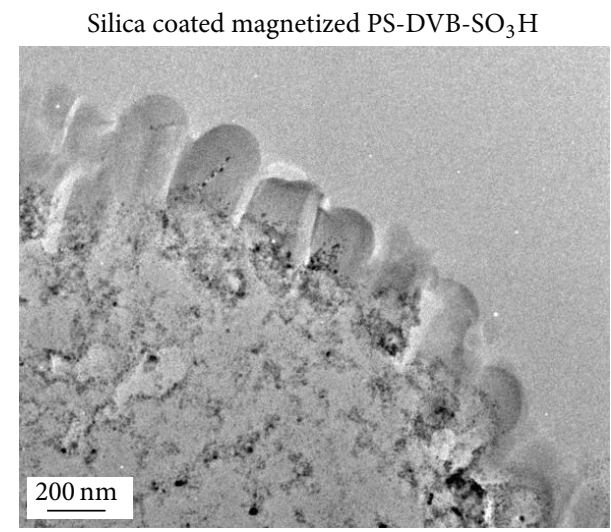

(f)

FIGURE 5: Silica coating of magnetized PS-DVB- $\mathrm{SO}_{3} \mathrm{H}$ microbeads. SEM images of magnetized PS-DVB-SO ${ }_{3} \mathrm{H}$ microbeads (a, c) before and (b, d) after silica coating at low magnification and high magnification. Cross-sectioned TEM images of (e) magnetized PS-DVB-SO ${ }_{3} \mathrm{H}$ and (f) silica coated magnetized PS-DVB- $\mathrm{SO}_{3} \mathrm{H}$ microbeads.

on the magnetic PS-DVB beads, the silica coated magnetic PS-DVB beads contained C (50.3\%), O (33.3\%), S (5.9\%), $\mathrm{Fe}(3.8 \%)$, and $\mathrm{Si}(6.7 \%)$ element as shown in Figure 6(c). Comparing to that of the magnetic PS-DVB beads, O element of the silica coated magnetic beads increased by $13 \%$, which is about two times Si element that demonstrated the presence of $\mathrm{SiO}_{2}$ on the surface of magnetic PS-DVB beads. Therefore, EDX data was consistent with TEM images and evidenced the successful deposition of silica shell on the magnetic PS-DVB beads surface.
To demonstrate the application of the silica coated magnetic beads, we used streptavidin-FITC as model biomolecules for bioseparation. Briefly, the silica coated magnetic beads surface was modified with amine group and conjugated with biotin through EDC/NHS. The fluorescent images were shown in Figures 6(d)-6(f). The fluorescence of the biotinylated silica coated magnetic beads without reaction with streptavidin-FITC was negligible comparing to the biotinylated silica coated magnetic beads treated with streptavidin-FITC as shown in Figure 6(e). Also we carried 


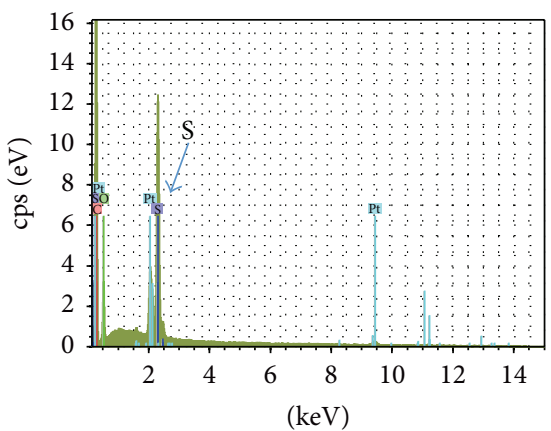

(a)

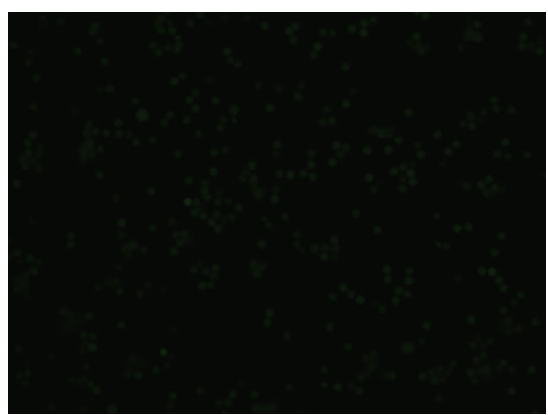

(d)

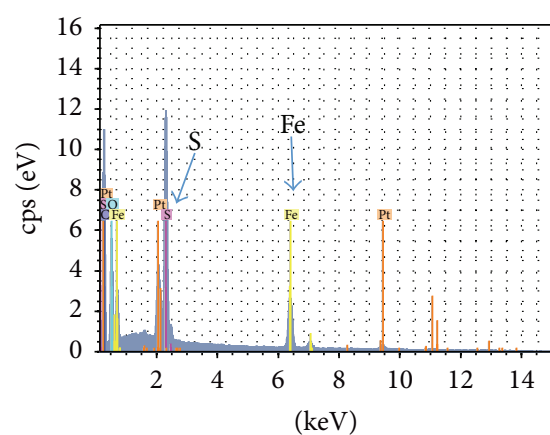

(b)

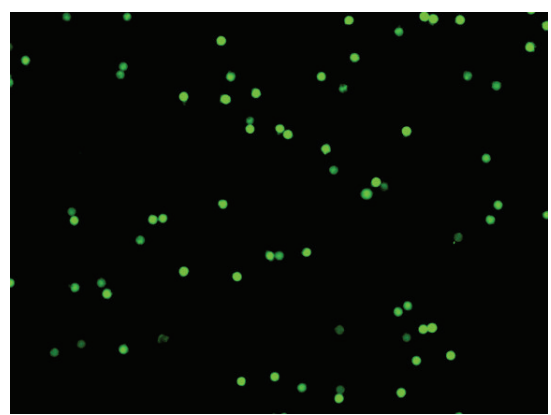

(e)

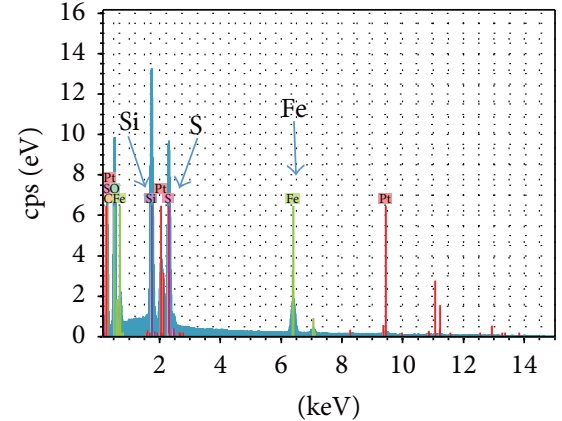

(c)

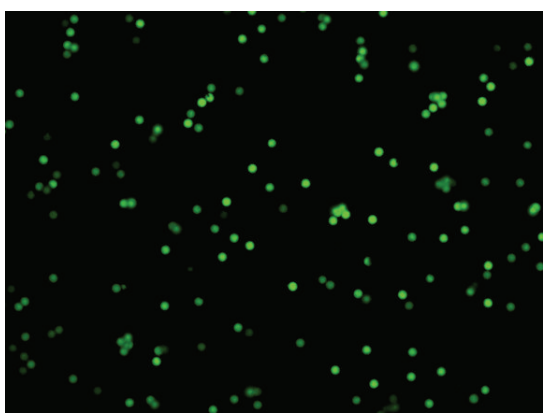

(f)

FIGURE 6: Chemical composition and bioapplication of silica coated magnetized PS-DVB- $\mathrm{SO}_{3} \mathrm{H}$ microbeads. EDX data of (a) PS-DVB-SO $\mathrm{H}_{3}$, (b) magnetized PS-DVB- $\mathrm{SO}_{3} \mathrm{H}$, and (c) silica coated magnetized PS-DVB- $\mathrm{SO}_{3} \mathrm{H}$ microbeads. Fluorescent images of silica coated magnetized PS-DVB- $\mathrm{SO}_{3} \mathrm{H}$ microbeads without (d) and with (e) streptavidin-FITC. (f) Nonspecific adsorption of streptavidin-FITC to silica coated magnetized PS-DVB- $\mathrm{SO}_{3} \mathrm{H}$ beads.

out the nonspecific binding test of silica coated magnetic beads without biotinylation and BSA blocking in Figure 6(f). Streptavidin-FITC easily adsorbed into the silica coated magnetic beads. Therefore, BSA blocking step played an important role in our protocol.

\section{Conclusions}

In conclusion, we successfully prepared monodisperse silica coated superparamagnetic magnetic beads using the macroporous polystyrene microbeads as template materials. The sulfonation of the porous PS-based beads and the subsequent magnetization successfully provided a means for preparing magnetic microbeads in straightforward and cost-effective way, which can be readily modified with silica shell as a watercompatible protective layer and surface modification site for various functional silane compounds. When used with flow cytometry or in a microfluidic system, the monodisperse magnetic microbeads may have great potentials for drug screening, medical diagnostics, and combinatorial chemical synthesis in more simple, convenient, and cost-effective way.
Abbreviations
FE-SEM: Field emission scanning electron microscopy
TEM: Transmission electron microscopy
FT-IR: Fourier transform infrared spectroscopy
TGA: Thermogravimetric analysis.

\section{Conflict of Interests}

The authors declare that there is no conflict of interests regarding the publication of this paper.

\section{Acknowledgments}

This work was supported by the National Research Foundation of Korea Grant funded by the Korean Government (NRF-2014S1A2A2027641) and by the Ministry of Science, ICT \& Future Planning (2014-A002-0065) and by the $2015 \mathrm{KU}$ Brain Pool of Konkuk University.

\section{References}

[1] C.-H. Chiou, Y.-Y. Huang, M.-H. Chiang, H.-H. Lee, and G.-B. Lee, "New magnetic tweezers for investigation of the mechanical properties of single DNA molecules," Nanotechnology, vol. 17, no. 5, pp. 1217-1224, 2006.

[2] C.-H. Chiou and G.-B. Lee, "A micromachined DNA manipulation platform for the stretching and rotation of a single DNA molecule," Journal of Micromechanics and Microengineering, vol. 15, no. 1, p. 109, 2005.

[3] L. Yi, G. Yu, and X. Chenjie, "Using magnetic nanoparticles to manipulate biological objects," Chinese Physics B, vol. 22, no. 9, Article ID 097503, 2013.

[4] E. Cho, M. N. Tahir, J. M. Choi, H. Kim, J. H. Yu, and S. Jung, "Novel magnetic nanoparticles coated by benzene- and $\beta$-cyclodextrin-bearing dextran, and the sorption of polycyclic 
aromatic hydrocarbon," Carbohydrate Polymers, vol. 133, pp. 221-228, 2015.

[5] Y. S. Park, C. Shin, H. S. Hwang et al., "In vitro generation of functional dendritic cells differentiated from CD34 negative cells isolated from human umbilical cord blood," Cell Biology International, vol. 39, no. 9, pp. 1080-1086, 2015.

[6] S. Kyeong, C. Jeong, H. Kang et al., "Double-layer magnetic nanoparticle-embedded silica particles for efficient bioseparation," PLoS ONE, vol. 10, no. 11, Article ID e0143727, 2015.

[7] M. Lee, Y. L. Kang, W. Y. Rho et al., "Preparation of plasmonic magnetic nanoparticles and their light scattering properties," RSC Advances, vol. 5, no. 27, pp. 21050-21053, 2015.

[8] S. Kyeong, C. Jeong, H. Y. Kim et al., "Fabrication of monodispersed silica-coated quantum dot-assembled magnetic nanoparticles," RSC Advances, vol. 5, no. 41, pp. 32072-32077, 2015.

[9] H. Mok and M. Q. Zhang, "Superparamagnetic iron oxide nanoparticle-based delivery systems for biotherapeutics," Expert Opinion on Drug Delivery, vol. 10, no. 1, pp. 73-87, 2013.

[10] J.-Y. Hyeon, J.-W. Chon, I.-S. Choi, C. Park, D.-E. Kim, and K.-H. Seo, "Development of RNA aptamers for detection of Salmonella Enteritidis," Journal of Microbiological Methods, vol. 89, no. 1, pp. 79-82, 2012.

[11] V. N. Morozov and T. Y. Morozova, "Active bead-linked immunoassay on protein microarrays," Analytica Chimica Acta, vol. 564, no. 1, pp. 40-52, 2006.

[12] Z. Jiang, J. Llandro, T. Mitrelias, and J. A. C. Bland, "An integrated microfluidic cell for detection, manipulation, and sorting of single micron-sized magnetic beads," Journal of Applied Physics, vol. 99, no. 8, Article ID 08S105, 2006.

[13] Y. Sun, Y. Bai, D. Song, X. Li, L. Wang, and H. Zhang, "Design and performances of immunoassay based on SPR biosensor with magnetic microbeads," Biosensors and Bioelectronics, vol. 23, no. 4, pp. 473-478, 2007.

[14] J. Broomberg, S. Gélinas, J. A. Finch, and Z. Xu, "Review of magnetic carrier technologies for metal ion removal," Magnetic and Electrical Separation, vol. 9, no. 3, pp. 169-188, 1999.

[15] T. R. Sathe, A. Agrawal, and S. Nie, "Mesoporous silica beads embedded with semiconductor quantum dots and iron oxide nanocrystals: dual-function microcarriers for optical encoding and magnetic separation," Analytical Chemistry, vol. 78, no. 16, pp. 5627-5632, 2006.

[16] R. F. H. Dekker, "Immobilization of a lactase onto a magnetic support by covalent attachment to polyethyleneimineglutaraldehyde- activated magnetite," Applied Biochemistry and Biotechnology, vol. 22, no. 3, pp. 289-310, 1989.

[17] C. H. Lochmuller and L. S. Wigman, "Affinity separations in magnetically stabilized fluidized beds: synthesis and performance of packing materials," Separation Science and Technology, vol. 22, no. 11, pp. 2111-2125, 1987.

[18] Y. Pan, X. Du, F. Zhao, and B. Xu, "Magnetic nanoparticles for the manipulation of proteins and cells," Chemical Society Reviews, vol. 41, no. 7, pp. 2912-2942, 2012.

[19] J. Bao, W. Chen, T. Liu et al., "Bifunctional $\mathrm{Au}-\mathrm{Fe}_{3} \mathrm{O}_{4}$ nanoparticles for protein separation,” ACS Nano, vol. 1, no. 4, pp. 293-298, 2007.

[20] Y. Zhang, W. Ma, D. Li, M. Yu, J. Guo, and C. Wang, "Benzoboroxole-functionalized magnetic core/shell microspheres for highly specific enrichment of glycoproteins under physiological conditions," Small, vol. 10, no. 7, pp. 1379-1386, 2014.
[21] J. Kim, H. S. Kim, N. Lee et al., "Multifunctional uniform nanoparticles composed of a magnetite nanocrystal core and a mesoporous silica shell for magnetic resonance and fluorescence imaging and for drug delivery," Angewandte ChemieInternational Edition, vol. 47, no. 44, pp. 8438-8441, 2008.

[22] B. Luo, S. Xu, A. Luo et al., "Mesoporous biocompatible and acid-degradable magnetic colloidal nanocrystal clusters with sustainable stability and high hydrophobic drug loading capacity," ACS Nano, vol. 5, no. 2, pp. 1428-1435, 2011.

[23] D. Li, J. Tang, C. Wei et al., "Doxorubicin-conjugated mesoporous magnetic colloidal nanocrystal clusters stabilized by polysaccharide as a smart anticancer drug vehicle," Small, vol. 8, no. 17, pp. 2690-2697, 2012.

[24] A. Chandna, D. Batra, S. Kakar, and R. Singh, "A review on target drug delivery: magnetic microspheres," Journal of Acute Disease, vol. 2, no. 3, pp. 189-195, 2013.

[25] D. Wang, J. He, N. Rosenzweig, and Z. Rosenzweig, "Superparamagnetic $\mathrm{Fe}_{2} \mathrm{O}_{3}$ beads-CdSe/ZnS quantum dots core-shell nanocomposite particles for cell separation," Nano Letters, vol. 4, no. 3, pp. 409-413, 2004.

[26] B.-H. Jun, M. S. Noh, J. Kim et al., "Multifunctional silverembedded magnetic nanoparticles as SERS nanoprobes and their applications," Small, vol. 6, no. 1, pp. 119-125, 2010.

[27] J. Gao, W. Zhang, P. Huang, B. Zhang, X. Zhang, and B. Xu, "Intracellular spatial control of fluorescent magnetic nanoparticles," Journal of the American Chemical Society, vol. 130, no. 12, pp. 3710-3711, 2008.

[28] J. Kim, Y. Piao, and T. Hyeon, "Multifunctional nanostructured materials for multimodal imaging, and simultaneous imaging and therapy," Chemical Society Reviews, vol. 38, no. 2, pp. 372390, 2009.

[29] H.-S. Cho, Z. Dong, G. M. Pauletti et al., "Fluorescent, superparamagnetic nanospheres for drug storage, targeting, and imaging: a multifunctional nanocarrier system for cancer diagnosis and treatment," ACS Nano, vol. 4, no. 9, pp. 5398$5404,2010$.

[30] E.-Q. Song, J. Hu, C.-Y. Wen et al., "Fluorescent-magneticbiotargeting multifunctional nanobioprobes for detecting and isolating multiple types of tumor cells," ACS Nano, vol. 5, no. 2, pp. 761-770, 2011.

[31] T. Alefantis, P. Grewal, J. Ashton, A. S. Khan, J. J. Valdes, and V. G. Del Vecchio, "A rapid and sensitive magnetic bead-based immunoassay for the detection of staphylococcal enterotoxin B for high-through put screening," Molecular and Cellular Probes, vol. 18, no. 6, pp. 379-382, 2004.

[32] J. Lundeberg, B. Pettersson, and M. Uhlén, "Direct DNA sequencing of PCR products using magnetic beads," in PCR Sequencing Protocols, R. Rapley, Ed., pp. 57-66, Springer, New York, NY, USA, 1996.

[33] S.-Q. Gu, Y.-X. Zhang, Y. Zhu, W.-B. Du, B. Yao, and Q. Fang, "Multifunctional picoliter droplet manipulation platform and its application in single cell analysis," Analytical chemistry, vol. 83, no. 19, pp. 7570-7576, 2011.

[34] T.-J. Yoon, K. N. Yu, E. Kim et al., "Specific targeting, cell sorting, and bioimaging with smart magnetic silica core-shell nanomaterials," Small, vol. 2, no. 2, pp. 209-215, 2006.

[35] J.-S. Choi, Y.-W. Jun, S.-I. Yeon, H. C. Kim, J.-S. Shin, and J. Cheon, "Biocompatible heterostructured nanoparticles for multimodal biological detection," Journal of the American Chemical Society, vol. 128, no. 50, pp. 15982-15983, 2006. 
[36] C.-W. Lu, Y. Hung, J.-K. Hsiao et al., "Bifunctional magnetic silica nanoparticles for highly efficient human stem cell labeling," Nano Letters, vol. 7, no. 1, pp. 149-154, 2007.

[37] J. Kim, S. Park, J. E. Lee et al., "Designed fabrication of multifunctional magnetic gold nanoshells and their application to magnetic resonance imaging and photothermal therapy," Angewandte Chemie-International Edition, vol. 45, no. 46, pp. 7754-7758, 2006.

[38] G. L. Liu, Y. Lu, J. Kim, J. C. Doll, and L. P. Lee, "Magnetic nanocrescents as controllable surface-enhanced raman scattering nanoprobes for biomolecular imaging," Advanced Materials, vol. 17, no. 22, pp. 2683-2688, 2005.

[39] P. A. Mosier-Boss and S. H. Lieberman, "Surface-enhanced raman spectroscopy substrate composed of chemically modified gold colloid particles immobilized on magnetic microparticles," Analytical Chemistry, vol. 77, no. 4, pp. 1031-1037, 2005.

[40] S. H. Choi, Y. N. Ko, K. Y. Jung, and Y. C. Kang, "Macroporous $\mathrm{Fe}_{3} \mathrm{O}_{4}$ /carbon composite microspheres with a short $\mathrm{Li}^{+}$ diffusion pathway for the fast charge/discharge of lithium ion batteries," Chemistry - A European Journal, vol. 20, no. 35, pp. 11078-11083, 2014.

[41] K. Deepankumar, S. P. Nadarajan, S. Mathew et al., "Engineering transaminase for stability enhancement and site-specific immobilization through multiple noncanonical amino acids incorporation," ChemCatChem, vol. 7, no. 3, pp. 417-421, 2015.

[42] M. S. Ramasamy, S. S. Mahapatra, and J. W. Cho, "Functionalization of graphene with self-doped conducting polypyrrole by click coupling," Journal of Colloid and Interface Science, vol. 455, pp. 63-70, 2015.

[43] M. Luqman, J.-W. Lee, K.-K. Moon, and Y.-T. Yoo, "Sulfonated polystyrene-based ionic polymer-metal composite (IPMC) actuator," Journal of Industrial and Engineering Chemistry, vol. 17, no. 1, pp. 49-55, 2011.

[44] B.-H. Jun, M. S. Noh, G. Kim et al., "Protein separation and identification using magnetic beads encoded with surfaceenhanced Raman spectroscopy," Analytical Biochemistry, vol. 391, no. 1, pp. 24-30, 2009.

[45] B. H. Jun, G. Kim, S. Jeong et al., "Silica core-based SurfaceEnhanced Raman Scattering (SERS) tag: advances in multifunctional SERS nanoprobes for bioimaging and targeting of biomarkers," Bulletin of the Korean Chemical Society, vol. 36, no. 3, pp. 963-978, 2015.

[46] W. Zhao, J. Gu, L. Zhang, H. Chen, and J. Shi, "Fabrication of uniform magnetic nanocomposite spheres with a magnetic core/mesoporous silica shell structure," Journal of the American Chemical Society, vol. 127, no. 25, pp. 8916-8917, 2005.

[47] M. S. Noh, B.-H. Jun, S. Kim et al., "Magnetic surface-enhanced Raman spectroscopic (M-SERS) dots for the identification of bronchioalveolar stem cells in normal and lung cancer mice," Biomaterials, vol. 30, no. 23-24, pp. 3915-3925, 2009.

[48] J.-H. Kim, J.-S. Kim, H. Choi et al., "Nanoparticle probes with surface enhanced Raman spectroscopic tags for cellular cancer targeting," Analytical Chemistry, vol. 78, no. 19, pp. 6967-6973, 2006.

[49] J. Ugelstad, "Swelling capacity of aqueous dispersions of oligomer and polymer substances and mixtures thereof," Die Makromolekulare Chemie, vol. 179, no. 3, pp. 815-817, 1978.

[50] T. K. Chambers and J. S. Fritz, "Effect of polystyrenedivinylbenzene resin sulfonation on solute retention in highperformance liquid chromatography," Journal of Chromatography A, vol. 797, no. 1-2, pp. 139-147, 1998.
[51] F. Schoebrechts, E. Merciny, and G. Duyckaerts, "Contribution de la chromatographie en phase liquide à haute performance à la séparation des lanthanides trivalents sur résine cationique en présence d’edta. I. Synthèse et propriétés générales des résines pelliculaires polystyrène-divinylbenzǹe sulfonates," Journal of Chromatography A, vol. 174, no. 2, pp. 351-360, 1979.

[52] A. Klingenberg and A. Seubert, "Comparison of silica-based and polymer-based cation exchangers for the ion chromatographic separation of transition metals," Journal of Chromatography $A$, vol. 640, no. 1-2, pp. 167-178, 1993.

[53] T. Hargitai, P. Reinholdsson, B. Törnell, and R. Isaksson, "Functionalized polymer particles for chiral separation," Journal of Chromatography A, vol. 630, no. 1-2, pp. 79-94, 1993.

[54] R. A. Weiss, A. Sen, C. L. Willis, and L. A. Pottick, "Block copolymer ionomers: 1. Synthesis and physical properties of sulphonated poly(styrene-ethylene/butylene-styrene)," Polymer, vol. 32, no. 10, pp. 1867-1874, 1991. 

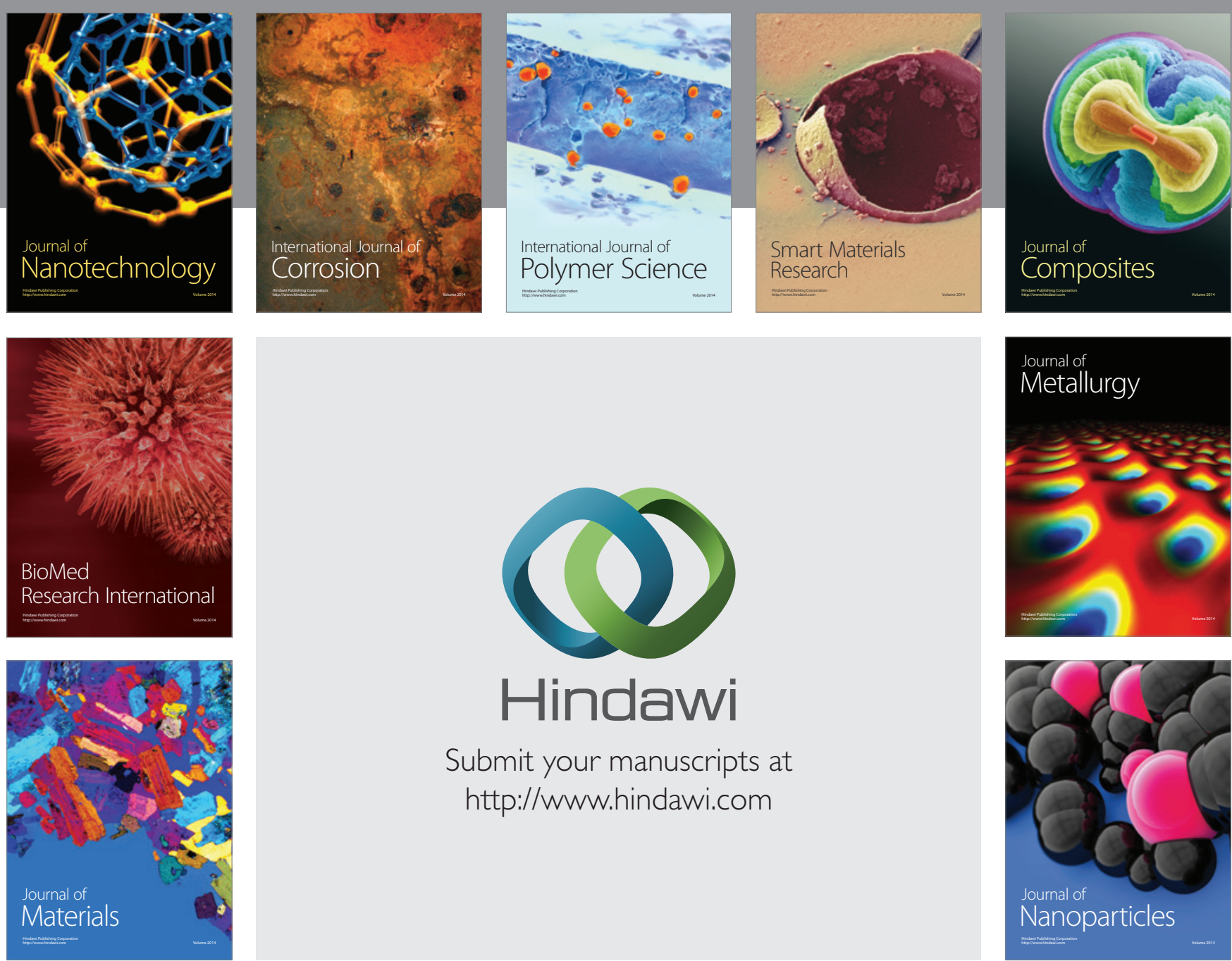

\section{Hindawi}

Submit your manuscripts at

http://www.hindawi.com

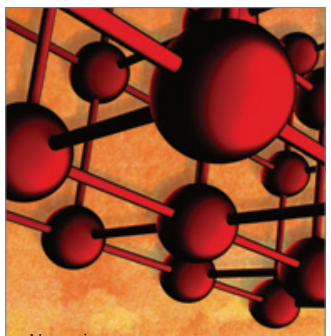

Materials Science and Engineering
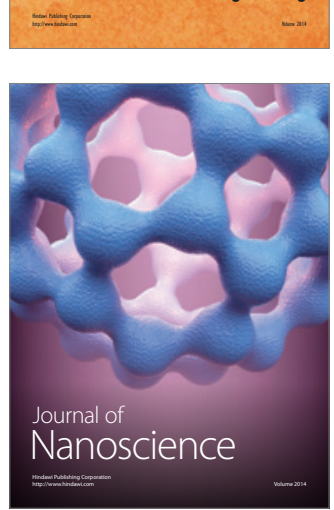
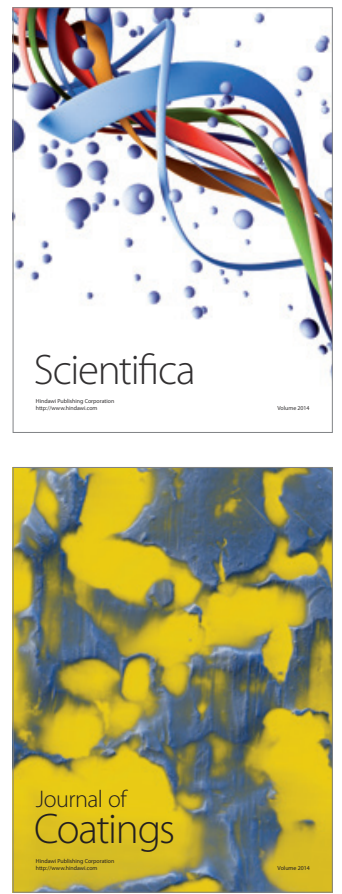
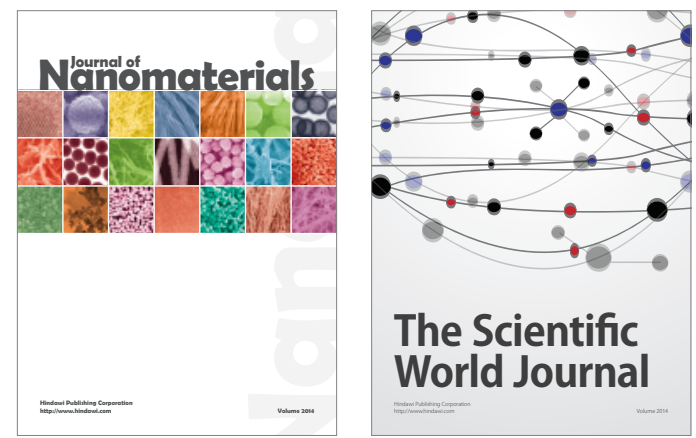

The Scientific World Journal
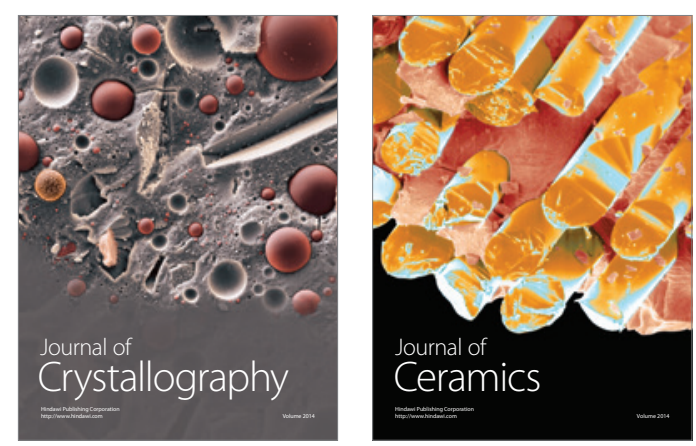
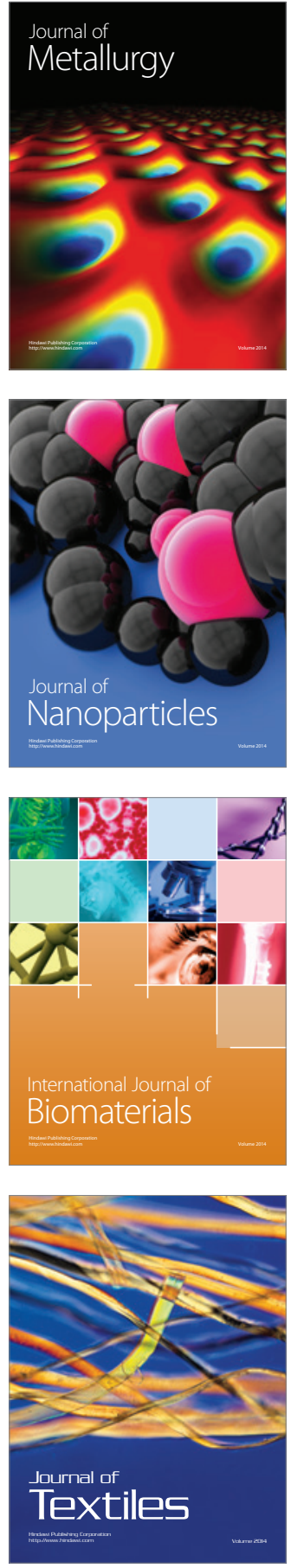